\title{
Object Detection using Geometric Invariant Moment
}

\author{
${ }^{1}$ Mohamed Rizon, ${ }^{2}$ Haniza Yazid, ${ }^{2}$ Puteh Saad, ${ }^{2}$ Ali Yeon Md Shakaff, ${ }^{2}$ Abdul Rahman Saad, \\ ${ }^{3}$ Mohd Rozailan Mamat, ${ }^{1}$ Sazali Yaacob, ${ }^{1}$ Hazri Desa and ${ }^{1}$ M. Karthigayan \\ ${ }^{1}$ School of Mechatronics Engineering \\ ${ }^{2}$ School of Computer and Communication Engineering \\ Kolej Universiti Kejuruteraan Utara Malaysia, Jalan Kangar-Arau 02600 Jejawi, Perlis, Malaysia \\ ${ }^{3}$ Terengganu Advanced Technical Institute (TATI), Jalan Panchor, Teluk Kalong \\ 24000 Kemaman, Terengganu, Malaysia
}

\begin{abstract}
Geometric moment invariant produces a set of feature vectors that are invariant under shifting, scaling and rotation. The technique is widely used to extract the global features for pattern recognition due to its discrimination power and robustness. In this paper, moment invariant is used to identify the object from the captured image using the first invariant (Ø1). The recognition rate for this technique is $90 \%$ after the image undergoes suitable processing and segmentation process.
\end{abstract}

Key words: Geometric invariant moment, object detection

\section{INTRODUCTION}

Moment invariants are important shape descriptors in computer vision. There are two types of shape descriptors: contour-based shape descriptors and region-based shape descriptors. Regular moment invariants are one of the most popular and widely used contour-based shape descriptors is a set of derived by $\mathrm{Hu}^{[1]}$. In this study, a computer vision system recognizing objects in captured images is established using Geometric Moment (GM).

In this experiment, the coconut is used throughout this research as the object of interest. Coconut is known in scientific as cocos nucifera and is a member of Family Arecaceae (palm family). Coconut is an important plant in the lives and economies of people in South East Asia like Burma, Indonesia, Philippines and Malaysia. In Malaysia, coconut is planted either for personal use or commercialize. There are varieties of commercial coconut that are planted in Malaysia such as Malayan Tall (MT), Malayan yellow Dwarf (MYD), Malayan Green Dwarf (MGD), Rennel Tall, Malayan Red Dwarf (MRD) and Pandan. Coconut provides almost all necessities of life like food, drink, oil, medicine, timber, thatch, mats, fuel and domestic utensils. For these, it has been called the "tree of heaven" and "tree of life". A 40 year old palm typically attains a height of $20-22 \mathrm{~m}$ and an 80 year old palm may attain a height of $35-40 \mathrm{~m}$. Due to the increasing usage of coconut, new method had to be explored to assist the coconut gripping process. It helps to pluck the coconut from a tree using image processing techniques and it will be faster, easier and convenient than the manual plucking.
There are several problems in detecting and recognizing the coconut in the image such as the target object is obscured due to the presence of the other object which can interfere with recognition process such as the fronds.

The moment based technique was successfully applied in trademark identification ${ }^{[2]}$, insect identification $^{[3,4]}$ use geometric invariant moment for pattern recognition. The main contribution of this work consists of using GM to recognize objects in captured images.

Proposed system: The image with 100x100 pixels was used through out this paper. PGM is used as the input to the proposed recognition process. Figure 1 shows the block diagram of the recognition process.

The input image has to come across several steps before the GM is performed. The image is enhanced using histogram equalization in preprocessing step and edge detection in segmentation step. Histogram equalization tends to increase the contrast of the image and produced a better result. Then, the image segmentation is carried out. The edge detection is employed to perform segmentation. Various edge detection methods have been applied for different application. Among them, Sobel edge detector is employed to the image. Canny gives thin edge that can cause false recognition between the coconut and the fronds. After the segmentation process, the image file is saved in .raw format and the GM is performed.

Geometric invariant moment: Geometric moment invariant was first introduced by $\mathrm{Hu}^{[1]}$. It was derived from the theory of algebraic invariant.

\footnotetext{
Corresponding Author: $\quad$ Mohamed Rizon, School of Mechatronic Engineering, Kolej Universiti Kejuruteraan Utara Malaysia, Jalan Kangar-Arau, 02600 Jejawi, Perlis, Malaysia, Tel: +60-4-9798335, Fax: +60-4-9798334
} 
GM technique is chosen to extract image features since the features generated are Rotation Scale Translation (RST)-invariant. Geometric Moment (G.M) was successfully applied in aircraft identification, texture classification and radar images to optical images matching ${ }^{[5]}$.

Two-dimensional moments of a digitally sampled $M \times M$ image that has gray function $f(x, y),(x, y=0, \ldots$ $. M-1)$ is given as,

$$
\begin{aligned}
& m_{p q}=\sum_{x=0}^{x=M-1} \sum_{y=0}^{y=M-1}(x)^{p} \cdot(y)^{q} f(x, y) \\
& p, q=0,1,2,3 \ldots
\end{aligned}
$$

The moments $f(x, y)$ translated by an amount $(a$, b), are defined as,

$\mu_{p q}=\sum_{x} \sum_{y}(x+a)^{p} \cdot(y+b)^{q} f(x, y)$

Thus the central moments $m_{p q}^{\prime}$ or $\mu_{p q}$ can be computed from (2) on substituting $\mathrm{a}=-\bar{x}$ and $\mathrm{b}=-\bar{y}$ as,

$$
\begin{aligned}
& \bar{x}=\frac{m_{10}}{m_{00}} \text { and } \bar{y}=\frac{m_{01}}{m_{00}}, \\
& \mu_{p q}=\sum_{x} \sum_{y}(x-\bar{x})^{p} \cdot(y-\bar{y})^{q} f(x, y)
\end{aligned}
$$

When a scaling normalization is applied the central moments change as,

$$
\eta_{p q}=\frac{\mu_{p q}}{\mu_{00}{ }^{\gamma}}, \gamma=[(p+q) / 2]+1
$$

In particular, $\mathrm{Hu}$ defines seven values, computed by normalizing central moments through order three, that are invariant to object scale, position and orientation. In terms of the central moments, the seven moments are given as below:

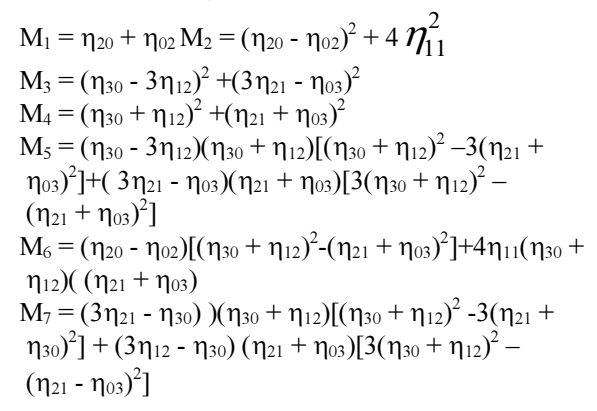

\section{RESULTS AND DISCUSSION}

The captured images for coconut and fronds were used to get the range of the invariant. The invariant feature vectors are as shown in the Table 1 and 2 .

From the Table 1 and 2, the coconut invariants for $\varnothing 1$ are in between 0.19 to 0.22 . The invariant $\varnothing 1$ for fronds is higher in between 0.24-0.36. For Ø2 onwards the invariant are insignificant. 50 images were used to
Table 1: Invariant feature vectors for coconut images

\begin{tabular}{lllll}
\hline & $\varnothing 1$ & $\varnothing 2$ & $\varnothing 3$ & $\varnothing 4$ \\
\hline Img_1 & 0.196626 & 0.000087 & 0.000050 & 0.000358 \\
Img_2 & 0.190880 & 0.000357 & 0.000008 & 0.000249 \\
Img_3 & 0.219196 & 0.000044 & 0.000013 & 0.000856 \\
Img_4 & 0.200170 & 0.000220 & 0.000086 & 0.001275 \\
Img_5 & 0.206258 & 0.000279 & 0.000002 & 0.000065 \\
Img_6 & 0.217532 & 0.000193 & 0.000036 & 0.003542 \\
Img_7 & 0.204132 & 0.000147 & 0.000026 & 0.000610 \\
Img_8 & 0.191695 & 0.000208 & 0.000008 & 0.000367 \\
Img_9 & 0.214011 & 0.000468 & 0.000002 & 0.000117 \\
Img_10 & 0.205873 & 0.001147 & 0.000088 & 0.003488 \\
\hline
\end{tabular}

Table 2: Invariant feature vectors for fronds images

\begin{tabular}{lllll}
\hline & $\varnothing 1$ & $\varnothing 2$ & $\varnothing 3$ & $\varnothing 4$ \\
\hline Img_1 & 0.261663 & 0.001267 & 0.000103 & 0.000050 \\
Img_2 & 0.339352 & 0.000089 & 0.000202 & 0.001565 \\
Img_3 & 0.324584 & 0.001538 & 0.000100 & 0.000193 \\
Img_4 & 0.332118 & 0.000302 & 0.000133 & 0.000233 \\
Img_5 & 0.285786 & 0.000386 & 0.000782 & 0.001561 \\
Img_6 & 0.278236 & 0.001206 & 0.000399 & 0.002175 \\
Img_7 & 0.251440 & 0.000059 & 0.000179 & 0.000080 \\
Img_8 & 0.245287 & 0.000115 & 0.000270 & 0.000723 \\
Img_9 & 0.284985 & 0.000543 & 0.000161 & 0.001754 \\
Img_10 & 0.351478 & 0.000180 & 0.000354 & 0.001718 \\
\hline
\end{tabular}

Table 3: Successful images and Ø1 invariant

\begin{tabular}{lc}
\hline Image & $\varnothing 1$ \\
\hline Image Figure 2 (a) & 0.197026 \\
Image Figure 2 (b) & 0.209452 \\
Image Figure 2 (c) & 0.218346 \\
Image Figure 2 (d) & 0.199167 \\
\hline
\end{tabular}

\begin{tabular}{lc} 
Table 4: Failure images and Ø1 invariant & \\
\hline Image & $\varnothing 1$ \\
\hline Image Figure 3 (a) & 0.209812 \\
Image Figure 3 (b) & 0.203113 \\
Image Figure 3 (c) & 0.187191 \\
Image Figure 3 (d) & 0.237937 \\
\hline
\end{tabular}

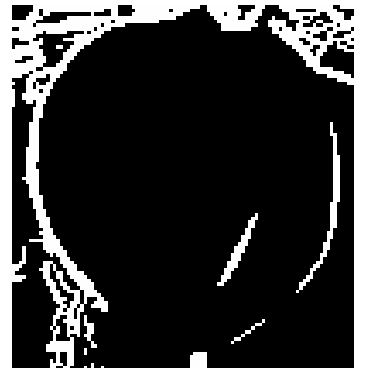

(a)

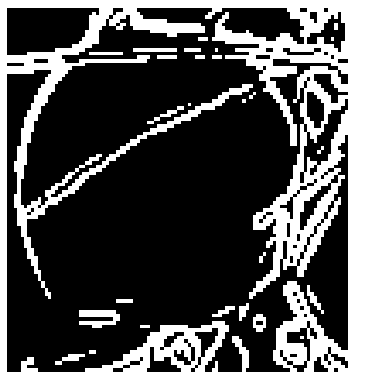

(b)

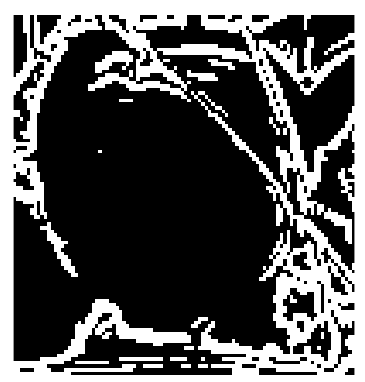

(c)

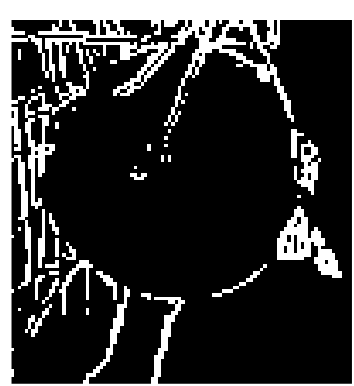

(d)
Fig. 2: Successful recognition of coconut images (a-d) 


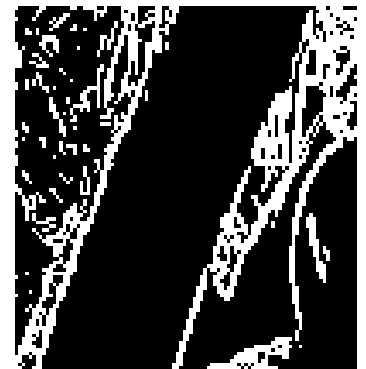

(a)

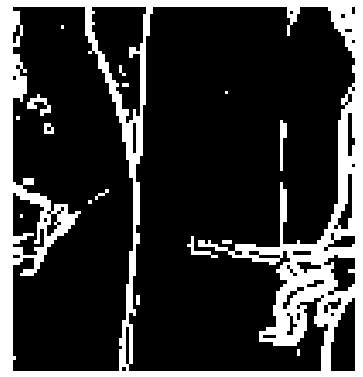

(b)

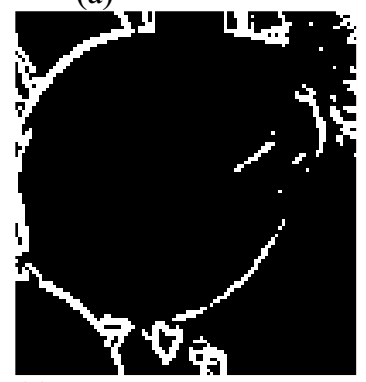

(c)

Fig. 3: Failure recognition of coconut images (a-d)

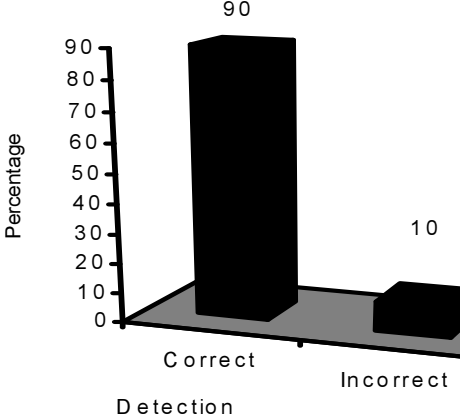

Fig. 4: Graph of correct and incorrect recognition after using Sobel edge detector

70

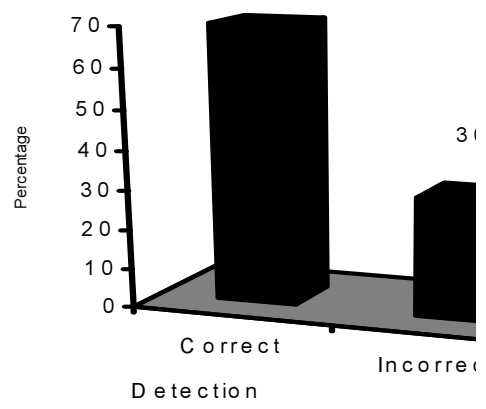

Fig. 5: Graph of correct and incorrect recognition after using Canny edge detector

evaluate the proposed technique. From 50 captured images, $90 \%$ gives positive result. The examples of the successful recognition are shown in Fig. 2. Some of the invariant are failed to recognize the object as shown in Fig. 3.

From the range that was analyzed, 50 images were tried to the proposed technique after using Sobel and
Canny edge detector in the segmentation process. The results are shown in Fig. 4 and 5.

GM can identify the presence of certain object in the captured image. From Table 1, the invariants for the $\varnothing 1$ are in some ranges $(0.19$ to 0.22$)$. But for $\varnothing 2$ onwards the invariants/features are insignificant. Hence, the identification of the coconut object in this research only used the $\varnothing 1$ invariant values. In ${ }^{[2]}$, the invariants from $\varnothing 1$ to $\varnothing 4$ are used in detecting the trademark images. Thus, the nature of the image used has a substantial effect on the feature values.

Some error may occur in detecting the coconut images. The error occurred when the invariant of the fronds images are quite similar to the coconut that is in between 0.19 to 0.22 . Meanwhile, some of the coconuts images are failed to detect as the coconut because of the invariant are out of the range. This problem occurred because the coconut image is too small and consist a lot of fronds.

The graphs show the identification using Sobel and Canny edge detector in segmentation process before using the GM. From the graph in Fig. 4 and 5, using Sobel is better rather than Canny. Sobel gives $90 \%$ successful recognition meanwhile Canny gives $70 \%$. Canny is too sensitive to detect the edge that effect the invariant in detection the object of interest.

\section{CONCLUSION}

This study presents the GM in identifying the object in the images. Sobel edge detection is used in the segmentation process before using the GM. Sobel tends to give a better result than Canny. From the finding obtained, only $\varnothing 1$ invariant is used in identifying the object. The invariant for the interest object is in the range of 0.19 to 0.22 .

\section{ACKNOWLEDGEMENT}

The authors wish to thank Kolej Universiti Kejuruteraan Utara Malaysia for their supports throughout this research.

\section{REFERENCES}

1. Hu, M.K., 1962. Visual pattern recognition by moments invariants. IRE Trans. Information Theory, 8: 179- 87.

2. Saad, P., 2004. Feature extraction of trademark images using geometric invariant moment and zernike momenta comparison. Chiang Mai J. Sci., 31: 217-222.

3. Yaakob, S.N., P. Saad and A.H. Abdullah, 2005. Insert recognition using fuzzy ARTMAP. Proc. Intl. Conf. Robotics, Vision, Information and Signal Processing ROVISP2005, pp: 679-684.

4. Paschalakis, S. and. P. Lee, 1999. Pattern recognition in grey level images using moment based invariant features, Image Processing and Its Application. Seventh Intl. Conf. (Conf. Publ. No. 46: 245-249.

5. Khotanzad, A., and Y.H. Hong, 1990. Invariant image recognition by zernike moments. IEEE Trans. Pattern Analysis and Machine Intelligence, 12: 489-497. 